\title{
Myeloid sarcoma of maxillary sinus
}

\author{
Simi C. M. ${ }^{1, *}$, K. R. Leenadevi², Boben Thomas ${ }^{3}$ \\ ${ }^{1,3}$ Consultant, ${ }^{2}$ Senior Consultant, Dept. of Pathology, Kerala Institute of Medical Sciences, Thiruvananthapuram, \\ Kerala, India
}

*Corresponding Author:

Email: ajusim@gmail.com

\begin{abstract}
We report a case of isolated myeloid sarcoma (MS) of maxillary sinus in a patient who had no evidence of leukemia at the time of diagnosis of MS. A 39 year old healthy woman presented with right cheek swelling of one year duration. CT scan of paranasal sinuses showed an illdefined, heterogenously enhancing soft tissue density lesion in relation to right maxilla. CT findings suggested the possibility of inflammatory phlegmon with osteomyelitis. Biopsy showed a malignant round cell neoplasm which on immunohistochemistry was confirmed to be myeloid sarcoma. On follow up, one month later her peripheral blood and bone marrow showed features of Acute Myeloid Leukemia which was confirmed to be AML- M2 on Flow cytometry. The diagnosis of myeloid sarcoma has always been difficult especially when it is presenting in an unusual location in a non leukemic patient.
\end{abstract}

Keywords: Acute Myeloid Leukemia, Maxillary sinus, Myeloid sarcoma.

Received: $30^{\text {th }}$ April, 2017

\section{Introduction}

Myeloid sarcoma is a tumor mass consisting of myeloid blasts with or without maturation occurring at an anatomical site other than the bone marrow ${ }^{1}$. Almost every site of body can be involved. Frequently affected sites are skin, lymphnode, bone, soft tissue and testis. ${ }^{2}$ Isolated myeloid sarcoma of paranasal sinuses are rare. ${ }^{7}$ Diagnosis tends to be difficult especially if the tumor does not occur in the context of AML and manifests in a very rare site. We are presenting such a rare case.

\section{Case History}

A 39 year old female presented to our dental department complaining of right cheek swelling of one year duration. On examination she had a hard well defined swelling measuring $3 \times 2 \mathrm{~cm}$ in relation to right maxilla obliterating the vestibule and extending to infraorbital margin. Routine laboratory investigations showed Haemoglobin$11.5 \mathrm{gm} / \mathrm{dl}$, Total count- $8400 / \mathrm{cmm}$, Platelet count- 2.88Lakhs. Differential count was within normal limits. CT scan of paranasal sinuses done elsewhere showed an illdefined, heterogenously enhancing, soft tissue density lesion measuring $3.5 \times 1.7 \times 2.8 \mathrm{~cm}$ in the right maxillary sinus. CT findings suggested the possibility of inflammatory phlegmon with osteomyelitis and advised biopsy to rule out malignancy. Incisional biopsy of the lesion was done. Macroscopy showed multiple grey white irregular tissue fragments aggregate measuring about
$1.4 \times 1 \times 0.4 \mathrm{cms}$. Microscopy showed a diffusely infiltrating neoplasm arranged in diffuse sheets and cords separated by hyalinised fibrous stroma and infiltrating walls of vessel and fat lobules (Fig. 1). Indian file pattern was seen in some areas. The cells were small to medium sized with vesicular nucleus, prominent nucleolus and moderate amount of eosinophilic cytoplasm. A report was given as Malignant round cell neoplasm and advised immunohistochemistry for further categorization. Our differentials were Lymphoma and undifferentiated carcinoma. A remote possibility of myeloid sarcoma was also considered as the morphology was not typical of the above conditions and as the tumor showed an indian file pattern. In the first panel of Immunohistochemistry, we included Pan CK, CD 20, $\mathrm{CD} \mathrm{3,} \mathrm{CD} \mathrm{5,} \mathrm{and} \mathrm{myeloperoxidase} \mathrm{(MPO).}$ The tumor cells were positive for MPO (Fig. 2) and were negative for CD 20, CD 3, CD 5, and Pan CK. In the second panel we added CD 117, CD 43, CD 34(Fig. 3) to support the diagnosis and to know the blastic nature and CD 68 to rule out monocytic differentiation. The tumor cells were positive for CD 117, CD 43, CD 34 and negative for $\mathrm{CD}$ 68. With these immunohistochemistry findings, a diagnosis of myeloid sarcoma was made. We advised oncology consultation, peripheral blood and bone marrow evaluation. 


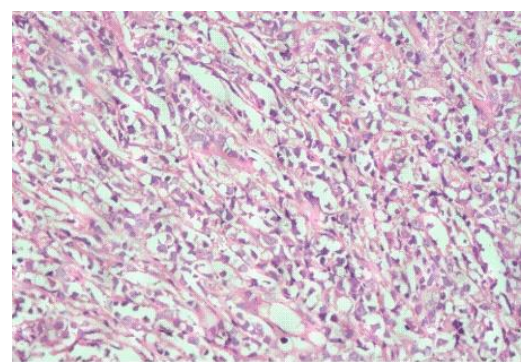

Fig. 1: Small to medium sized tumor cells infiltrating in cords and strands (H \& E X 200)

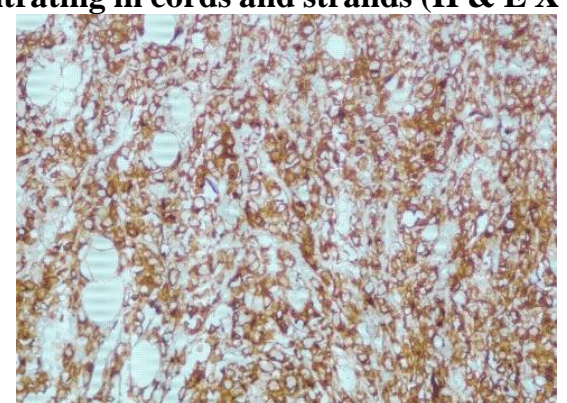

Fig. 2: Immunohistochemistry for myeloperoxidase (MPO) showing strong positivity in the tumor cells (original magnification $X$ 200)

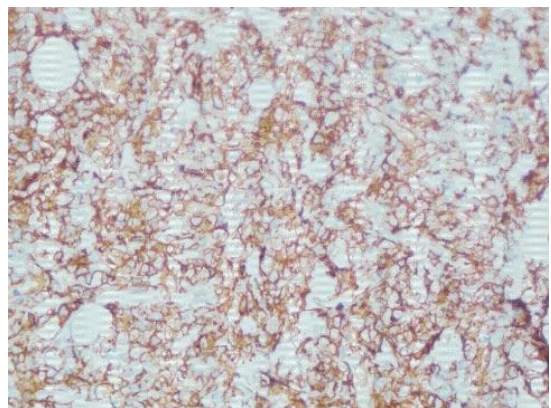

Fig. 3: CD34 Immunohistochemical stain showing strong positivity in the tumor cells (original magnification $X$ 200)

One month later we received her peripheral smear and bone marrow aspirate for evaluation. At the time of bone marrow evaluation, the haemogram showed: Haemoglobin-10.4 gm/dl, total count- $5500 / \mathrm{cmm}$ and platelet count -1.67 Lakhs. Peripheral smear and bone marrow aspirate showed many blasts with increased N:C ratio, fine chromatin, two to three nucleoli. Some of these cells had Auer rods in the cytoplasm. A diagnosis of Acute myeloid leukemia was given. Flow cytometry showed gated leucocytes in blast region highly positive for CD33, CD34, CD45, CD117, HLADR, MPO, and moderately positive for CD13. Flow cytometry confirmed the diagnosis as AML -M2. Cytogenetics showed $\mathrm{t}$ $(8 ; 21)(\mathrm{q} 22 ; \mathrm{q} 22)$. Inversion $16(\mathrm{p} 13 ; \mathrm{q} 22)$ and $\mathrm{t}$ $(15,17)(\mathrm{q} 21 ; \mathrm{q} 22)$ was not detected.
The patient was put on Cytarabine based chemotherapy. She completed chemotherapy. Her cheek swelling disappeared. Her peripheral blood counts are within normal limits. She is on regular follow up and is doing well.

\section{Discussion}

Myeloid sarcoma may occur denovo, precede AML or coincide with AML or occur after the diagnosis of AML. ${ }^{2,8}$ It may represent an acute blastic transformation of myelodysplastic syndrome (MDS), myeloproliferative neoplasm (MPN) and MDS/MPN. ${ }^{2}$ MS may also be the initial manifestation of relapse in a patient with previously diagnosed AML. ${ }^{2}$

Paranasal sinuses are very rare sites for myeloid sarcoma especially MS preceding AML. ${ }^{6,9}$ Our case highlights such a rare presentation. Clinically our case presented as cheek swelling and radiologically interpreted as inflammatory lesion. The clinical diagnosis of myeloid sarcoma is difficult and the definitive diagnosis can only be made after histological and immunohistochemical analysis. In cases lacking a history of any known hematological abnormality, it may be misdiagnosed since MS can mimic lymphoma or undifferentiated carcinoma on histology. A correct diagnosis requires a high index of suspicion. A diagnosis of MS should be thought of in any unusual, odd-looking or difficult to classify tumor and in tumors showing a prominent Indian file pattern of infiltration of stroma. ${ }^{1}$ Currently the most sensitive and specific immunohistochemical marker is Myeloperoxidase. ${ }^{1}$ For poorly differentiated MS, Myeloperoxidase may be negative and the diagnosis can be supported by positive staining for CD 117 and CD 34. ${ }^{1}$ For monocytic type of MS, Myeloperoxidase is negative, where as CD 68 and CD 163 are usually positive. ${ }^{1}$ In our case, biopsy done to exclude malignancy, helped to arrive at a diagnosis of MS. This was followed by peripheral blood and bone marrow evaluation. Our case is isolated MS preceding AML.

Myeloid sarcoma has been described in association with a variety of chromosomal abnormalities like $\mathrm{t}(8: 21)$ and inv $16 .^{5}$ Our case showed $\mathrm{t}(8: 21)$. Some studies have shown that interaction of some chemokine receptors (CXCR4, CCR5, CXCR7 and CX3CR1) results in homing and retention of the blasts in other sites. ${ }^{3}$ The current treatment recommendation for isolated Myeloid sarcoma and Myeloid sarcoma occuring in AML patients is AML- type chemotherapy. ${ }^{3}$ Diagnosis of Myeloid sarcoma in a nonleukemic patient in an unusual site is often a challenge for pathologist. 


\section{References}

1. J.K.C. Chan. Tumors of lymphoreticular system. In: Fletcher DM. Diagnostic Histopathology of Tumors. $2^{\text {nd }}$ ed.Churchill Livinstone;2000.p.1099245.

2. Swerdlow, Campo, Harris, Jaffe, Pileri, Stein et al editors-WHO Classification of Tumours of Haematopoietic and Lymphoid Tissues. $4^{\text {th }}$ ed.IARC.Lyon,2008.110-41.

3. Batia Avni and Maya Korean-Michowitz. Myeloid Sarcoma: Current approach and therapeutic options. Therapeutic Advances in Hematology;2011;309-16

4. Cristina Campidelli, Claudio Agostinelli, Richard Stitson, Stefano. Myeloid sarcoma: Extramedullary manifestation of myeloid disorders. American journal of clinical pathology 2009;132:426-37

5. Alexiev, Wang, Ning, Chumsri, Gojo, Rodgers et al- Myeloid sarcomas: A histologic, immunohistochemical, and cytogenetic study. Diagnostic Pathology 2007;2:42-8.

6. Kai-Di Mei, Yung-song Lin, Shih- Lun Chang. Myeloid sarcoma of cheek and the maxillary sinus regions. Journal of the Chinese Medical Association 2013, 76,235-8

7. Chi-Kuang young, Tuan- Jen Fang, Yushin-Hung, Wen-Yu Chuang, Ta-Jen Lee. Isolated Myeloid sarcoma of the Maxillary sinus. A case report and review of Literature. J Cytol Histol 2014,5:3

8. Cristina Campidelli, Claudio Agostinelli, Richard Stitson, Stefano A. Pileri. Myeloid Sarcoma: Extramedullary Manifestation of Myeloid Disorders,Am J Clin Pathol 2009;132:426-37.

9. Jane Zhou, Diana Bell, L. Jeffrey Medeiros, Myeloid Sarcoma of the Head and Neck Region. Arch Pathol Lab Med. 2013;137:1560-8. 